\title{
Offshore wind farm layout optimization using particle swarm optimization
}

\author{
Ajit C. Pillai ${ }^{1,2}$ (1) $\cdot$ John Chick $^{3} \cdot$ Lars Johanning $^{2} \cdot$ Mahdi Khorasanchi ${ }^{4}$
}

Received: 18 April 2017 / Accepted: 3 January 2018 / Published online: 23 January 2018

(c) The Author(s) 2018. This article is an open access publication

\begin{abstract}
This article explores the application of a wind farm layout optimization framework using a particle swarm optimizer to three benchmark test cases. The developed framework introduces an increased level of detail characterizing the impact that the wind farm layout can have on the levelized cost of energy by modelling the wind farm's electrical infrastructure, annual energy production, and cost as functions of the wind farm layout. Using this framework, this paper explores the application of a particle swarm optimizer to the wind farm layout optimization problem considering three different levels of wind farm constraint faced by modern wind farm developers. The particle swarm optimizer is found to yield improvements in the layout with respect to the levelized cost of energy for the three benchmark cases when compared to two past studies. This highlights both applicability of the particle swarm optimizer to the problem and the ways in which a wind farm developer could make use of the present framework in the development and design of future wind farms.
\end{abstract}

Keywords Offshore wind $\cdot$ Layout optimization $\cdot$ Particle swarm optimization $\cdot$ Wind farm design

\section{Introduction}

As the world transitions to a more sustainable energy sector, wind energy and in particular offshore wind farms represent a significant means for reducing the greenhouse gas emissions of electricity generation. As the offshore wind energy industry has grown, both the size of wind farms and the size of individual turbines have grown significantly. Wind

\footnotetext{
Ajit C. Pillai

a.pillai@exeter.ac.uk

John Chick

john.chick@ed.ac.uk

Lars Johanning

1.johanning@exeter.ac.uk

Mahdi Khorasanchi

khorasanchi@sharif.edu
}

1 Industrial Doctorate Centre for Offshore Renewable Energy (IDCORE), The University of Edinburgh, Edinburgh, UK

2 Present Address: Renewable Energy Group, University of Exeter, Penryn, UK

3 Institute for Energy Systems, The University of Edinburgh, Edinburgh, UK

4 Department of Mechanical Engineering, Sharif University of Technology, Tehran, Iran farms now represent much larger projects both in terms of the area they cover and their generational capacity than the early projects of the past decade. With many projects currently in development, it has become increasingly important to ensure that these wind farms are designed in a sophisticated manner making use of the available area as efficiently as possible.

To meet this need, tools have been developed exploring the optimal placement of wind turbines, offshore substations, and intra-array cables within an offshore wind farm. The original work in wind farm layout optimization done by Mosetti et al. (1994) laid the ground work for this field introducing a general approach that following work has continued to utilize. This approach includes the assessment of both the energy produced by a wind farm and the cost of the wind farm over the lifetime of the project. More recent work in the field of offshore wind farm layout optimization has explored the applicability of different optimization algorithms as well as the inclusion of additional constraints and more detailed cost functions that a developer may face. The most frequent optimization algorithm applied to the wind farm layout optimization problem has been the genetic algorithm with several studies exploring its applicability to the problem as posed by Mosetti et al. (1994) and to more complex extensions (Chen et al. 2013; Couto et al. 2013; Elkinton 
2007; Elkinton et al. 2008; Geem and Hong 2013; Grady et al. 2005; Huang 2009; Mittal 2010; Shakoor et al. 2016; Zhang et al. 2014). In a similar vein, recent studies have also explored optimization algorithms such as viral based optimization (Ituarte-Villarreal and Espiritu 2011), pattern search (DuPont and Cagan 2012), mixed-integer linear programming (Fagerfjäll 2010), Monte Carlo method (Marmidis et al 2008), and random search (Feng and Shen 2015) applied to the wind farm layout optimization problem.

An optimization algorithm that has emerged as relevant to this problem and has frequently been deployed for variations on this problem is the particle swarm optimizer (PSO) (Chowdhury et al. 2012, 2013; Hou et al. 2017; Pookpunt and Ongsakul 2013; Wan et al. 2010a,b). These existing studies have included various considerations beyond the problem originally defined in the seminal work in the field by Mosetti et al. (1994) such as hub height variations, turbine capacity variations, and intra-array cable routing (Chowdhury et al. 2013; Feng et al. 2016; Hou et al. 2017). However, these have still not considered several elements that would be important to a real wind farm developer.

The present work, therefore, builds on the standard paradigm in wind farm layout optimization by considering not only the impact the wind farm layout has on the energy produced by the wind farm, but also the impact of layout design and turbine placement on the electrical infrastructure and the wind farm's lifetime costs. Extending the previous work in this field as well as that of the authors (Pillai et al. 2016b), the present work presents this optimization problem with the inclusion of three constraint sets of interest to wind farm developers and applies these to a series of benchmark cases in which the levelized cost of energy (LCOE), a single metric that considers the wind farm energy output and costs over the wind farm's lifetime, is used to compare layouts.

This paper introduces increased detail in the evaluation a wind farm layout as well as additional constraint levels that a developer will face in the design of a real offshore wind farm, thereby striving to capture the impacts the wind farm layout can have on the LCOE and explores the optimization of wind farm layouts using a cooperative population based metaheuristic optimization approach ${ }^{1}$, particle swarm optimization. This, therefore, involves returning to the key reference work by Mosetti et al. (1994) and Grady et al. (2005) and demonstrating that with the increased level of detail in the evaluation function and the three different constraint sets, a particle swarm optimizer is not only a relevant optimization algorithm, but is also capable of identifying improvements to the layouts regardless of the size of wind farm.

\footnotetext{
${ }^{1}$ A metaheuristic optimization approach is a general strategy that is applicable to a wide range of optimization problems by making few or no assumptions about the problem (Burke and Kendall 2013).
}

Section 2 introduces the approach of the wind farm layout optimization framework describing the components and the optimization algorithm deployed. Section 3 introduces the specific cases explored in this paper with the results presented in Sect. 4. Section 5 analyses these results before the conclusions of this study are summarized in Sect. 6.

\section{Approach}

In general, wind farm layout optimization requires two principal components, one for assessing the quality of a given wind farm layout and a second for altering the layouts in an effort to improve them. The standard paradigm for the optimization of wind farm layouts makes use of the LCOE for assessing the quality of the layout, integrating wind farm wake models and cost models in order to ascertain the LCOE for a given layout. In this application, lower LCOE values represent better layouts. The present methodology expands on the standard paradigm by including the electrical infrastructure as the initial step in the determination of the LCOE. The location of the offshore substations and the design of the intra-array cable network impacts both the annual energy production (AEP) and the costs and is, therefore, an important step in assessing the impact of changes to the turbine layout. The modular design of the approach, shown in Fig. 1, has allowed different wake, cost, and optimization algorithms to be implemented as part of the development of the tool. Prior to integration through the optimization algorithm, each of the components of the evaluation function have been independently validated (Pillai et al. 2014, 2015, 2016a). The optimization algorithm, the PSO in the present work, then makes use of the LCOE values in order to advise the next iteration of proposed layouts.

Existing offshore wind farms have generally been designed using simple spacing rules with turbines laid out along regular grids. Though this is the preferred approach from the perspective of search and rescue practitioners and helps to maintain navigational routes through the wind farm, it does limit the designs that a developer could deploy (NOREL

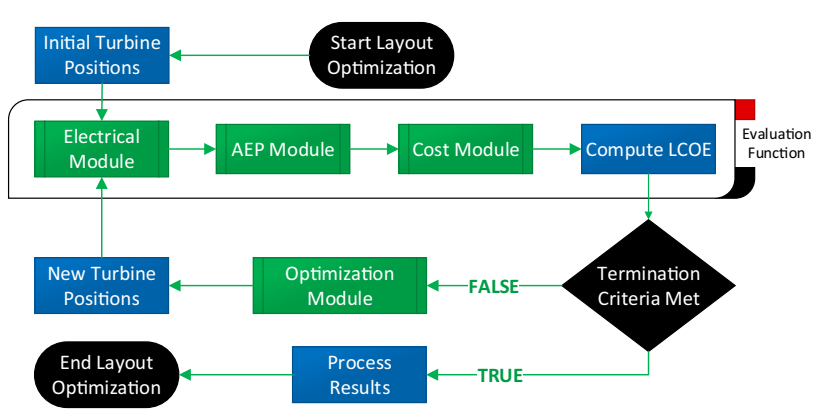

Fig. 1 Modular approach to wind farm layout optimization 
Group 2014). In order to explore the different levels of constraints under which wind farms are currently being designed, allowing greater flexibility to the wind farm developer, three constraint sets are implemented each requiring a different optimization problem to be implemented. Under these constraints, the wind turbine positions are either on a fixed grid defined by the optimizer, one of a set of pre-defined allowable turbine positions, or anywhere within the wind farm area that satisfies the seabed constraints. These varying degrees of constraint on the wind farm design represent the different approaches taken by European regulators in order to offer flexibility to the wind farm developers while still accounting for the interests and concerns of other marine stakeholders.

\subsection{Evaluation of LCOE}

As described, the wind farm layout optimization tool compares layouts on a basis of LCOE as this is a single metric which represents the cost effectiveness of a layout. The LCOE, measured in energy generation per unit cost, takes into account both the lifetime energy production of the wind farm and the lifetime costs of the project and is a common metric used by project developers to compare designs and competing projects. The energy production and costs are both discounted in order to represent the total lifetime energy production and lifetime costs in present value terms. In this way, the LCOE represents the ratio of the present value of the inputs to the present value of the outputs of the wind farm (Tegen et al. 2012, 2013).

$$
\mathrm{LCOE}=\frac{\sum_{t=1}^{n} \frac{C_{t}}{(1+r)^{t}}}{\sum_{t=1}^{n} \frac{\mathrm{AEP}_{t}}{(1+r)^{t}}}
$$

where $C_{t}$ is the total costs incurred in year $t, n$ is the project lifetime, $\mathrm{AEP}_{t}$, is the annual energy production in year $t$, and $r$ is the discount rate of the project.

\subsubsection{Electrical infrastructure design}

The first step in the evaluation of a layout as shown in Fig. 1 is the design of the necessary electrical infrastructure to support the given layout considering any seabed restrictions which may be present at the site. As the electrical infrastructure impacts the energy produced by the wind farm due to losses through the electrical system, and changes to the electrical infrastructure can impact the project costs, the inclusion of this step helps quantify the impact on the LCOE of changes to the wind farm layout. The methodology for this is described in greater detail by the authors in Pillai et al. (2015). The majority of existing wind farm layout optimization tools have not considered the impact of the turbine layout on either the intra-array cable collection networks or substation positions and the impact that these changes will have on the LCOE (Chen et al. 2013; Chowdhury et al. 2013; Couto et al. 2013; DuPont and Cagan 2012; Elkinton 2007; Elkinton et al. 2008; Geem and Hong 2013; Grady et al. 2005; Huang 2009; Ituarte-Villarreal and Espiritu 2011; Marmidis et al 2008; Mosetti et al. 1994; Réthoré et al. 2011; Shakoor et al. 2016; Zhang 2013; Zhang et al. 2014). The existing tools that have included this step in the optimization of a wind farm layout, have, however, omitted bathymetric constraints which a real-world developer would face (Feng et al. 2016; Hou et al. 2017). Furthermore, existing standalone tools have explored the optimization of the intra-array cable network for an offshore wind farm as an independent problem. These approaches have similarly, also not considered the irregular seabed exclusion areas for intra-array cables which arise from both bathymetric and regulatory constraints that the developer may face at sites. As these exclusion areas are often non-convex polygons in shape, their accurate inclusion in previous work has proven challenging (Bauer and Lysgaard 2015; Dutta and Overbye 2013; Lindahl et al. 2013; Rodrigues et al. 2016).

The optimization of the electrical infrastructure as developed in Pillai et al. (2015) uses of a series of heuristics and is, therefore, not guaranteed to identify the proven optimal solution; however, it has been found to identify good quality solutions in an acceptable runtime thereby representing a pragmatic approach to this real-world problem. This optimization process identifies not only the substation positions, and cable paths given the bathymetric constraints, but also the conductor sizes for each electrical cable in the network. This methodology to optimize the electrical infrastructure is shown in Algorithm 1.

The first step in this process is the determination of the substation positions by clustering the turbine positions. By making use of a modified clustering algorithm based on $k$-means++ (Arthur and Vassilvitskii 2007), the clustering process is capable of generating substation positions which adhere to the seabed constraints and their own capacity constraints while still minimizing the distance to the turbines. From here, a pathfinding algorithm is executed to generate the fully connected set of cable paths for the given turbine and substation positions. The pathfinding algorithm is used in order to consider the seabed obstacles which define where the cables cannot be placed. Using the accurate lengths of cables determined by the pathfinding algorithm, a capacitated minimum spanning tree (CMST) problem is formulated and solved using a commercial MILP solver, Gurobi (Gurobi Optimization Inc. 2015). The solution to the CMST identifies which of the cables should be deployed in the final network. In this way, the pathfinding step defines all the possible cables to consider and their accurate lengths, while the construction of the CMST selects which of these cables should be used 


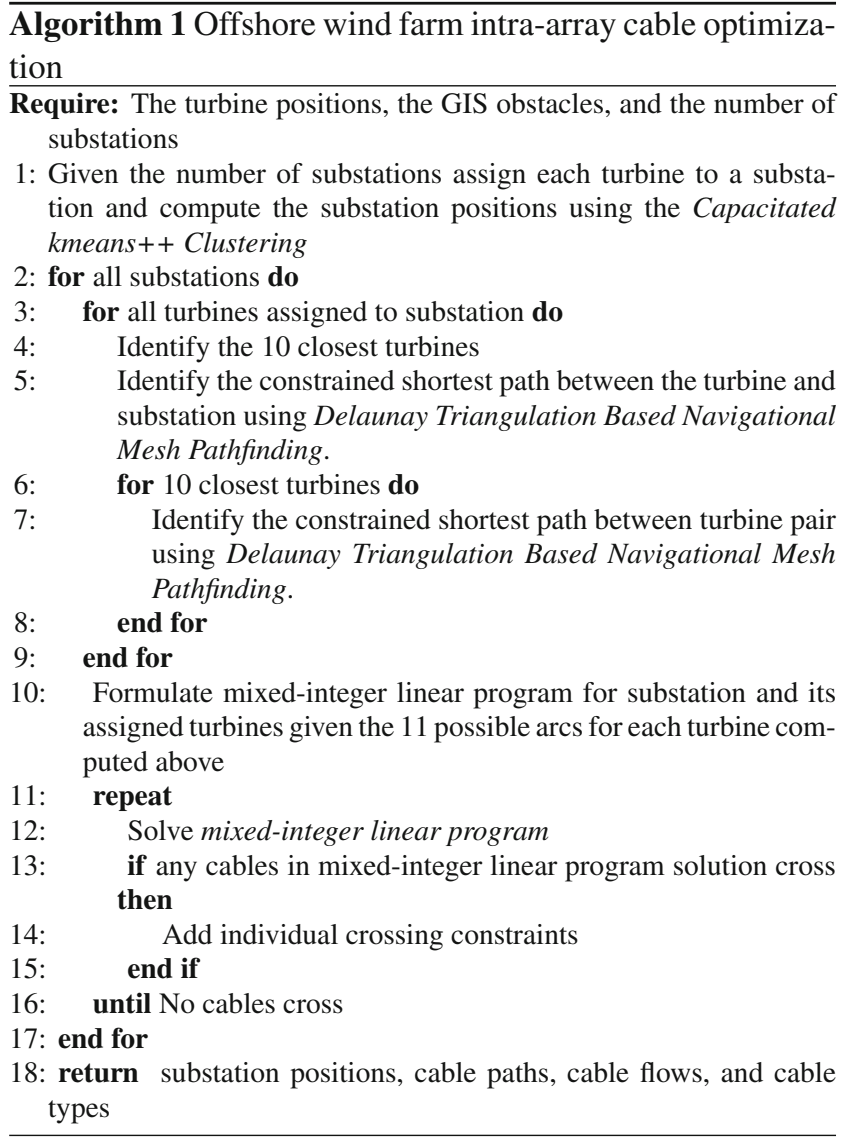

to minimize the cost of the infrastructure. Following this, the pathfinding algorithm is again deployed to determine the export cable path from each substation now considering the intra-array network as constraint regions to ensure that the export cable does not cross any of the intra-array cables.

Using this sub-tool, the electrical constraints of the cables and substations are not only taken into account, but seabed features dictating where this equipment cannot be placed are also considered. As intra-array cables can exceed $£ 500,000$ per $\mathrm{km}$ installed, it is important that the impact the wind farm layout has on the amount of cable needed is included in the assessment of the layout's cost (Gaillard 2015). Furthermore it is not uncommon for large offshore wind farms to be characterized by a number of constraint regions which can significantly impact the design of the intra-array collection network (Pillai et al. 2015).

\subsubsection{AEP estimation}

It is well understood that any device extracting energy from a natural flux has some impact on that flux. Wind turbines are no different, and directly behind an operating wind turbine, the air flow is affected due to the extraction of energy. In this region, known as the wake, the wind is characterized by reduced speeds and increased levels of turbulence compared to the conditions upstream of the turbine (Barthelmie et al. 2006, 2009; Makridis and Chick 2013; Renkema 2007). The layout of a wind farm can, therefore, have a major impact on the wind speeds that each individual wind turbine within the wind farm experiences and thereby the energy production of the farm as a whole. As a result of this, it is important for the wind turbine wakes to be accounted for both when estimating wind farm production figures and the LCOE of a given layout.

The calculation of the AEP within this tool is done using an industry standard analytic approach in which the wake losses are accounted for using the Larsen wake model (Larsen 1988). This model has been selected as validation using site data demonstrated that it represented a good compromise between computational intensity and accuracy (Gaumond et al. 2012; Pillai et al. 2014). The Jensen wake model used in previous layout optimization work has been found in validation studies to under-estimate the AEP and is, therefore, not as well suited for this work as the Larsen model (Gaumond et al. 2012).

To compute the AEP, each wind speed and direction combination are stepped through in turn. For each free wind speed and wind direction the analytic wake model is used to update each turbine's experienced wind speed based on the performance of all upwind turbines. From this, the wind turbine power curve is used to convert the incident wind speed to the energy generated under the given conditions. For each wind speed and direction combination, the energy losses through the electrical cable network are then computed based on each turbine's individual contribution to the AEP and the total wind farm contribution to AEP under the given free-stream wind speed and direction is updated. This total production for each wind speed and direction combination is then scaled by the probability of occurrence of this combination for the site in question before being added to the AEP.

$\mathrm{AEP}=8766 \times \sum_{d_{i}} \sum_{v_{i}} P\left(d_{i}, v_{i}\right) \times\left[E\left(d_{i}, v_{i}\right)-L\left(E\left(d_{i}, v_{i}\right)\right)\right]$

where $d_{i}$ is the wind direction; $v_{i}$ is the wind speed; $P\left(d_{i}, v_{i}\right)$ is the joint probability of $d_{i}$ and $v_{i} ; E\left(d_{i}, v_{i}\right)$ is the energy production for the wind farm for the combination of incident wind speed and direction considering the wake losses; and $L\left(E\left(d_{i}, v_{i}\right)\right)$ is the electrical losses associated with the wind speed and direction as a result of the intra-array cable network. These electrical losses are assessed using an IEC loss calculation based on IEC 60228 and IEC 60287 (IEC 2006a, b). This methodology is similar to that used by commercial tools such as WindFarmer and WindPRO which include both the losses due to wakes and within the intra- 
Table 1 Cost contribution to CAPEX and OPEX

\begin{tabular}{lllll}
\hline Cost element & CAPEX & OPEX & DECEX & Inclusion of layout \\
\hline Turbine supply & $\checkmark$ & - & - & Low \\
Turbine installation & $\checkmark$ & - & - & Medium \\
Foundation supply & $\checkmark$ & - & - & Medium \\
Foundation installation & $\checkmark$ & - & - & Medium \\
Intra-array cables & $\checkmark$ & - & - & High \\
Operations and maintenance (O\&M) & - & $\checkmark$ & - & Medium \\
Decommissioning & - & - & $\checkmark$ & Medium \\
Offshore transmission assets & $\checkmark$ & $\checkmark$ & - & Low \\
\hline
\end{tabular}

array cable network (DNV GL—Energy 2014; Thøgersen 2005).

\subsubsection{Cost assessment}

The final step in the evaluation of the LCOE as shown in Fig. 1 is the estimation of the costs over the lifetime of the project. Whereas previous tools have assumed a cost which scales with the number of turbines, the approach used in this tool seeks to more accurately capture the impact that the wind farm layout has on the lifetime costs. Layouts with the same number of turbines may, therefore, have different costs using this model as opposed to the cost model frequently deployed in layout optimization which represents the cost as a function of only the number of turbines.

The project costs are divided into eight principal cost centres with varying degrees of dependency to the wind farm layout as shown in Table 1. The capital expenditure (CAPEX) elements are incurred either in the construction stage of the project or in the case of decommissioning at the end of the project life and discounted appropriately while the operational expenditure (OPEX) elements are incurred in each year of operation following the construction period and prior to the decommissioning period. The decommissioning costs are categorized as decommissioning expenditure (DECEX) and are incurred at the end of life during the decommissioning period during which there is no OPEX incurred.

Each of these cost elements considers not only the turbine positions relative to one another, but also the turbine positions relative to the construction and O\&M ports, as well as the depth at each individual turbine's position. Relevant cost centres also consider the vessel parameters, cable parameters, and design parameters of the substations. The specific cost relationships have been developed in discussions with wind farm developers and suppliers in order to ensure that the costs are representative of the costs to be incurred by future projects in European waters and accurately capture the impact that the turbine layout can have on these costs.
Turbine supply The cost associated with the supply of the turbines is based entirely on a price per turbine supplied by turbine manufactures. This cost is, therefore, independent of the layout of the wind farm and factor only of the number of turbines or installed capacity of the wind farm.

Turbine installation Using market values for vessel costs and their capacities, the turbine installation costs are modelled by assessing the total amount of time required to install the turbines at their specific locations within the wind farm. This, therefore, includes the calculation of the time required for each installation operation, the travel time between turbines, and the travel time to and from the construction port. In order to determine the optimal vessel installation route, the turbines are clustered based on the capacity of the installation vessel, and for each cluster a shortest path is computed between the port, each turbine in the cluster, and the port again. This approach, therefore, accurately computes the distance that the vessel must travel over the installation process. From this, the total time is computed based on assumed weather availability and the costs computed based on the vessel and equipment day rates. The turbine layout, therefore, has a direct impact on the time needed to travel between turbine positions as well as to and from the port.

Foundation supply Foundation costs are found to be highly dependent on the site conditions where the foundation is to be installed. To account for this dependence, previous cost models have attempted a bottom up approach based on the soil characteristics at the installation site to model the costs. Unfortunately, this approach has proven difficult to validate for all foundation types (Elkinton 2007). For the present tool, therefore, a depth dependency has been developed from discussions with manufacturers and the specific soil conditions are not included. Detailed bathymetry of a site is, therefore, necessary in order to accurately estimate the variation in foundation supply costs as a function of the turbine layout. As the original cases defined by Mosetti et al. (1994) did not include bathymetric data, a constant depth has been assumed across the site. 
Foundation installation The foundation installation process like the turbine installation module is based on estimating the time needed to complete the operations and converting this time to a cost. Unlike the turbine installation though, this is modelled as three distinct phases which each uses a different vessel to complete.

Regardless of the foundation type (gravity-based, monopile, or jacket), some seabed preparation is necessary. For a gravity-based foundation this might be the necessary dredging and levelling of the seabed, while for monopiles and jackets this would more likely be pre-pilling works including surveying and drilling. After this step, the foundations will be installed as a separate operation following which some kind of scour protection will often be added. The installation of scour protection is again modelled as a separate step involving a different vessel from either the site preparation or foundation installation processes. In some conditions, the scour protection will not be necessary; however, for the time being the present model assumes that all turbines will require scour protection.

Intra-array cable costs The total horizontal length of intraarray cables required is computed from the intra-array cable optimization tool described earlier. This tool is described in detail in previous work by the authors (Pillai et al. 2015). This tool has the support for optimizing the layout for different cable cross-section sizes and, therefore, can output not only the total length of cable, but the horizontal lengths required for each segment and the required cross-section. From this, the intra-array cable cost module computes the necessary vertical cable and the necessary spare cable before computing the costs.

Following the calculation of the supply cost, the installation cost is computed in a similar manner to the turbine and foundation installation modules. This is done based on data available for cable trenching vessels and, therefore, assumes that all cables are trenched and buried.

Operations and maintenance The operations and maintenance costs are based on a tool developed by EDF Energy R\&D UK Centre which models the anticipated operations and maintenance cost of a project to vary with the projects distance from the operations and maintenance port and the capacity of the project. As this term is affected by distance of the wind farm to the operations and maintenance port, this too is affected by the layout. The operations and maintenance costs are classed as operational expenditure (OPEX) as these are incurred each year of operation as opposed to the preceding cost elements which are only incurred during the construction period and are, therefore, classed as CAPEX elements.
Decommissioning The decommissioning costs include the removal of the turbines and foundations. At the moment, it is unclear what will happen to the transmission and export cables at the end of a wind farm's life. The model, therefore, assumes that these cables are not removed at the time of decommissioning, but simply cut at the turbines and substation, leaving the buried lengths as they are. The decommissioning costs are, therefore, modelled similar to the installation processes with the time each vessel is required first computed before this is converted to a cost. Like the installation processes it is assumed that the vessels have some finite capacity and must return to the decommissioning port during the overall operation. The turbines and foundations are assumed to be decommissioned in separate steps requiring separate vessels. Like the installation phases, this term is, therefore, dependent on the turbine positions and is affected by the proposed layout.

Offshore transmission assets The final cost element of this cost model is the inclusion of the offshore transmission asset transfer fees. In the UK, the offshore substation, export cables, and onshore substation must be owned and operated by a separate company from the wind farm operator. Practically, therefore, most wind farm developers build these assets, and then transfer them to a transmission operator before commissioning the wind farm. As a result, only some of the CAPEX is incurred by the project, and the rest is incurred as a component of the transmission fee along with regionally based costs set by the network operator; in the UK this is National Grid. Both the CAPEX and OPEX components of the Offshore Transmission Owners' assets have been computed in discussion with National Grid and equipment manufacturers based on the capacity of the assets.

\subsection{Particle swarm optimization}

The particle swarm optimization algorithm is a populationbased metaheuristic based on the behaviour of flocking birds or shoaling fish (Eberhart and Kennedy 1995; Kennedy and Eberhart 1995). In this respect, the algorithm treats the candidate solutions as particles within a swarm which are exploring the search space cooperatively. Each particle (solution) changes its position in the search space between iterations based on a velocity vector defined by the knowledge of both the swarm's past position and the individual particle's historical positions within the search space. For iteration $i$ of the process, this velocity, $v$, for a given particle is given by:

$v_{i}=C_{1} \times v_{i-1}+C_{2} \times r_{1}\left(p-x_{i}\right)+C_{3} \times r_{2}\left(g-x_{i}\right)$, 
where $C_{1}, C_{2}$, and $C_{3}$ are coefficients representing the weighting of each of the contributors determined by tuning the PSO; $p$ is the best position that the particle has historically occupied within the search space; $g$ is the best position that any individual within the swarm as a whole has ever occupied; $x$ is the solution under consideration; and $r_{1}$ and $r_{2}$ are two random numbers between 0 and 1 selected using a uniform distribution. With this velocity the particle's position the next iteration is given by:

$x_{i+1}=x_{i}+v_{i}$

Once each particle's position is updated, the evaluation function is used to determine the corresponding LCOE for each of the proposed layouts. Each particle's historical best position $p$ is then updated if needed, and the best $p$ value is used to define $g$. These updated $p$ and $g$ values are needed in the determination of the updated particle velocities for the next iteration of the process.

Compared to the genetic algorithm or alternate metaheuristics which have been applied to the wind farm layout optimization problem, the PSO is of interest as in optimization benchmarking studies it has been found to find high-quality solutions in less time than a similar genetic algorithm (Eberhart et al. 2001; Hassan et al. 2005). Given the complexity of future wind farms, this is of interest to wind farm developers as the PSO could, therefore, identify better solutions than the industry standard approaches using commercial software tools thereby leading to more efficient wind farm layouts. Furthermore, whereas the genetic algorithm is seen as a competitive metaheuristic in which individual solutions compete for survival, the PSO fosters a cooperative environment where the individual solutions directly impact one another. In this way, all members of the swarm are made aware of the improvements found by each individual particle, using this information to inform their future movements within the search space.

The parameters of the present PSO are given in Table 2. Due to the available computational power, this study used a constant swarm size of 100 particles. In order to ensure that the velocity vector does not take a particle outside of the search space, a dynamic velocity clamping approach was used in which velocity limits are imposed in each direction based on the location of the particle. This is similar to the trajectory constriction approach described by Clerc and Kennedy (2002) and Van Den Bergh and Engelbrecht (2006). For the binary constraints described below, a binary implementation of the PSO in which all decision variables are binary variables is necessary. As the velocity in the binary implementation must correspond to a specific decision variable being either a 1 or a 0 , a velocity transfer function is required to convert the velocity for each decision variable into a probability that the decision variable should be a 1 .
Table 2 Particle swarm parameters

\begin{tabular}{ll}
\hline Parameter & Description \\
\hline Swarm size & 100 \\
Velocity clamping & Dynamic \\
$\begin{array}{l}\text { Velocity transfer function } \\
\quad \text { binary encoding) }\end{array}$ & $T(x)=\left|\frac{2}{\pi} \times \arctan \left(x \cdot \frac{\pi}{2}\right)\right|$ \\
$\begin{array}{l}\text { Neighbourhood topology } \\
\text { Stop criteria }\end{array}$ & Global (gBest) \\
$\begin{array}{l}\text { Maximum generations reached } \\
\text { No improvement over 50 generations }\end{array}$ & Diversity $<10 \%$ \\
\hline
\end{tabular}

In the original study by Mosetti et al. (1994), the wind farm area was discretized into 100 allowable turbine positions. The optimizer is, therefore, tasked with the selection of which of these positions to use for the deployed wind turbines. This, therefore, represents a constraint on the turbine placement and it would be expected that better layouts could be achieved if this constraint was relaxed. To explore this, three different constraints on the turbine placement are used in the present study:

1. Array constraints: The turbine positions are constrained to being on a regular grid with constant downwind and crosswind spacings. The decision variables of the optimization problem define the spacing and orientation of the regular grid of turbine positions with constant downwind and crosswind spacing throughout the site.

2. Binary constraints: The turbine positions are limited to being one of a predefined set of allowable turbine positions. For the present study, the wind farm area is discretized into 100 allowable turbine positions as defined (Mosetti et al. 1994) and the decision variables of the optimization problem are binary variables representing the presence of a turbine in a particular cell. This represents the case in which the wind farm developer, regulator, and stakeholders define a set of acceptable turbine positions and the wind farm is designed by selecting turbine positions form this set.

3. Continuous constraints: The turbine positions can be anywhere within the wind farm boundary that is technically feasible. The decision variables directly define the turbine coordinates and may, therefore, occupy any value within the wind farm area. This represents a situation in which the wind farm developer is free to design the wind farm as they see best limited only by the technical constraints of the site.

The three approaches represent different ways in which the problem can be defined all of which are used by wind farm developers to design and explore the available options in the 


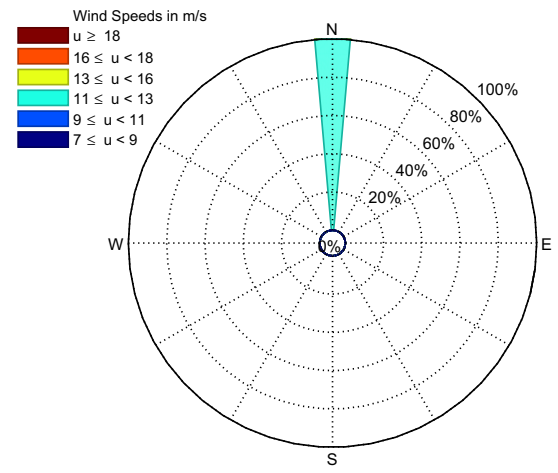

(a) Case 1

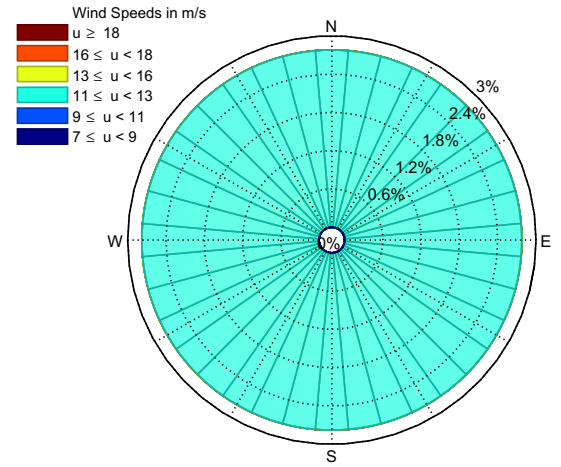

(b) Case 2

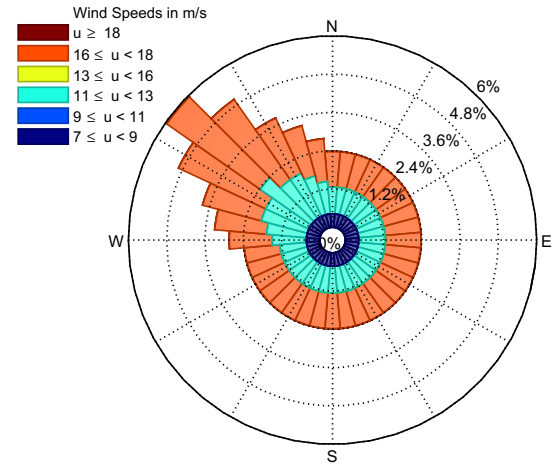

(c) Case 3

Fig. 2 Wind roses for the three different resource cases

design of an offshore wind farm. The array and binary constraint sets are of interest to a wind farm developer in regions where the regulator imposes some degree of symmetry as a result of navigational and search and rescue safety concerns (NOREL Group 2014). As the three constraint sets have fundamentally different degrees of complexity and represent different design spaces the optimizers were tuned individually for each of the problems in an attempt to maximize the performance though the same swarm size was used for all cases. Regardless of the placement constraints used, the technical seabed constraints such as the position of wrecks, unexploded ordnance, and the seabed slope are considered. For all three constraint sets, a minimum separation constraint is applied to ensure that turbines do not risk colliding and the wind farm boundary explicitly defines the limits of the wind farm.

\section{Definition of cases}

In the development of layout optimization tools three case studies have been defined by Mosetti et al. (1994). These three cases have been commonly used in order to evaluate the performance and demonstrate the capabilities of wind farm layout optimization tools. In order to demonstrate the capabilities of the present framework, which makes use of a more detailed layout evaluation function, the three cases are approached using the original constraints as well as under two different sets of relaxed constraints. Through this, the capabilities of the present framework using a PSO are highlighted.

The three cases all consider a $2 \mathrm{~km}$ by $2 \mathrm{~km}$ area in which turbines must be placed; however, they differ with regards to the wind resource. Case one considers a case of constant wind speed and constant wind direction in which the wind is constantly $12 \mathrm{~m} \mathrm{~s}^{-1}$ and from the $10^{\circ}$ sector centred on $0^{\circ}$. The second case is described as the case of constant wind speed and variable direction in which the wind is again constantly $12 \mathrm{~m} \mathrm{~s}^{-1}$, but now has an equal probability of blowing from any of the 36 discrete wind directions. Finally, the third case, the case of variable wind speed and direction, describes a case in which both the wind speed and wind direction are variable and most closely resembles a true wind farm. All three cases describe the resource using 36 discrete wind directions (Fig. 2) which are each used in the calculation of the AEP and the modelling of the wakes in the evaluation function. Validation studies of analytic wake models have found that these models are not necessarily more accurate when using narrower wind direction sectors, and discrete wind sectors of $10^{\circ}$ to $30^{\circ}$ in size should be used when deploying analytic wake models such as the Jensen or Larsen models (Gaumond et al. 2013; Pillai et al. 2014).

The original cases do not define the water depth nor are the locations of the relevant ports defined. In order to allow comparison with existing results for these case studies, the water depth has been assumed constant across the site and the ports have been placed far away relative to the size of the wind farm.

\section{Results}

In order to demonstrate the capabilities of the present framework using a PSO, the final layouts from the original study by Mosetti et al. (1994) and the final layouts from a subsequent study by Grady et al. (2005) are evaluated using the present evaluation function in order to offer a fair comparison to the new layouts proposed. These two studies used different numbers of turbines for each resource case and, therefore, cannot be directly compared to one another. Likewise, much of the literature has also allowed the number of turbines to vary thereby making direct comparisons challenging. In the present framework, the number of turbines is fixed, thereby allowing a direct comparison on the same number of tur- 


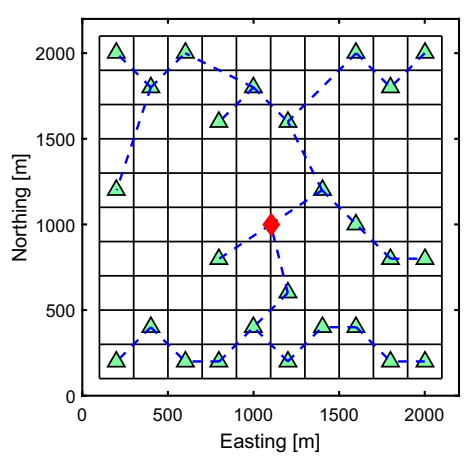

(a) Case 1 - 26 Turbines

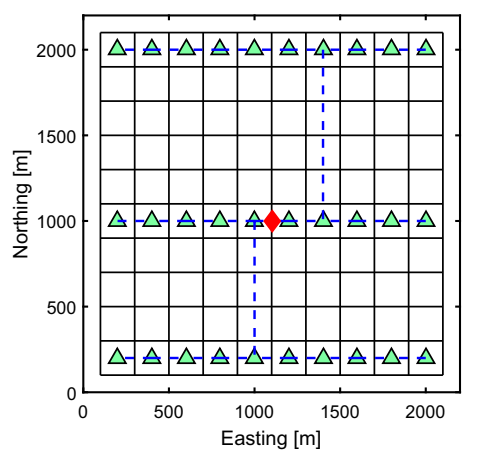

(d) Case 1 - 30 Turbines

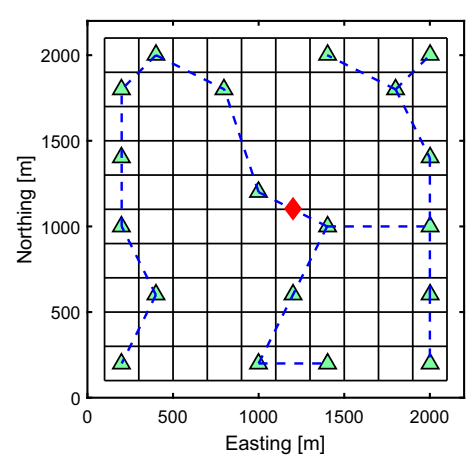

(b) Case 2 - 19 Turbines

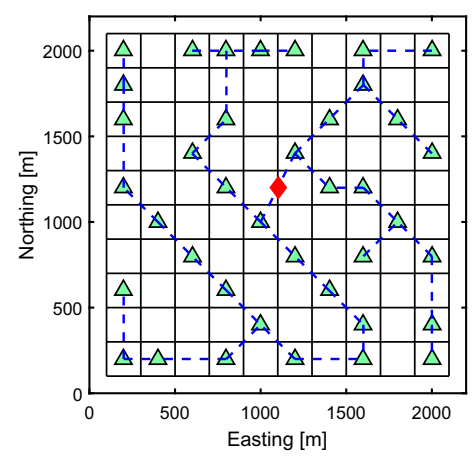

(e) Case 2 - 39 Turbines

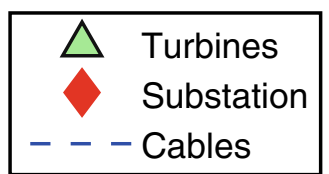

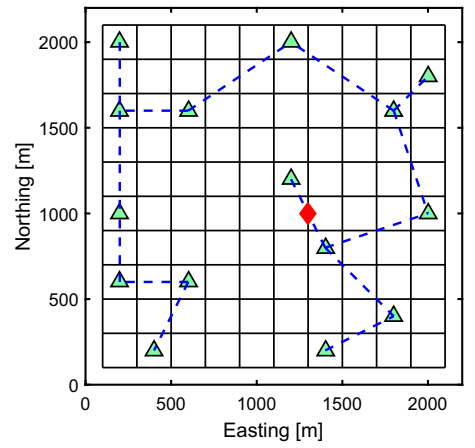

(c) Case 3 - 15 Turbines

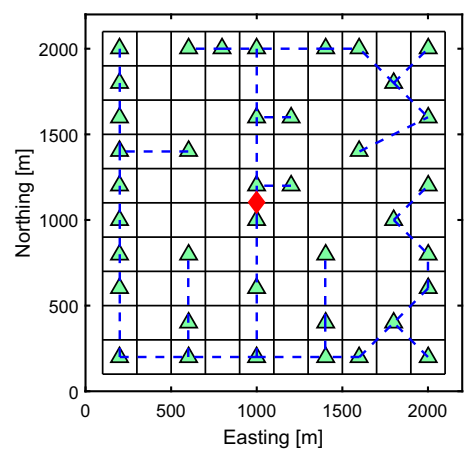

(f) Case 3 - 39 Turbines

Fig. 3 Original optimized layouts proposed by Mosetti et al. (1994) on the top row and Grady et al. (2005) on the bottom row for the three resource cases

bines against both the reference case study and the different constraint sets.

The original layouts produced in the studies by Mosetti et al. (1994) and Grady et al. (2005) for all three resource cases are shown in Fig. 3. The studies performed by Mosetti et al. (1994) and Grady et al. (2005) both allowed the number of turbines to vary and, therefore, for each of the resource cases, the two studies present different wind farm sizes. In the present study, each wind farm resource is executed with all three sets of constraints and at same the wind farm sizes as reported in the two past studies in order to fairly compare to the reference studies. The binary constraint set represents the most similar case to the problem originally defined by Mosetti et al. (1994); however, the present tool uses a fixed number of turbines, while the original studies allowed this to change. Each of the presented optimization results represents the converged results after a maximum of 100 generations. In general, less than 60 generations were required to reach the converged results presented.

\subsection{Case 1: constant wind speed, constant direction}

The results presented in Table 3 and Fig. 4 shows the outputs from re-evaluating the original layouts proposed in the previous studies (Grady et al. 2005; Mosetti et al. 1994) as well as the outputs from the execution of the PSO for this case. As the developed method uses the number of turbines as an input to the optimization process, it was necessary to execute the optimizer for two different wind farm sizes corresponding to the studies originally performed by Mosetti et al. (1994) and Grady et al. (2005), respectively, allowing the results to be directly compared to these past studies (shown in Fig. 3a, d). As described above, each of the wind farm sizes was run with three different types of constraints corresponding to different requirements on the placement of the turbines.

From the results presented in Table 3 it can be observed that for both wind farm sizes, the PSO either finds improvements or the same solution proposed by the references cases regardless of which constraint set was used. Specifically, 
Table 3 Layout optimization results: constant wind speed, constant direction

\begin{tabular}{lllll}
\hline Study & $\begin{array}{l}\text { Number of } \\
\text { Turbines }\end{array}$ & Lifetime cost (£) & AEP (MWh) & LCOE (£/MWh) \\
\hline Mosetti et al. (1994) & 26 & $4.42 \times 10^{8}$ & $9.90 \times 10^{4}$ & 522.87 \\
Array constraints & 26 & $4.39 \times 10^{8}$ & $1.18 \times 10^{5}$ & $\mathbf{4 3 4 . 8 7}$ \\
Binary constraints & 26 & $4.41 \times 10^{8}$ & $1.01 \times 10^{5}$ & 510.46 \\
Continuous constraints & 26 & $4.42 \times 10^{8}$ & $1.16 \times 10^{5}$ & 447.18 \\
Grady et al. (2005) & 30 & $4.77 \times 10^{8}$ & $1.13 \times 10^{5}$ & 496.29 \\
Array constraints & 30 & $4.76 \times 10^{8}$ & $1.33 \times 10^{5}$ & $\mathbf{4 1 9 . 6 1}$ \\
Binary constraints & 30 & $4.77 \times 10^{8}$ & $1.13 \times 10^{5}$ & 496.29 \\
Continuous constraints & 30 & $4.78 \times 10^{8}$ & $1.33 \times 10^{5}$ & 421.64 \\
\hline
\end{tabular}

Bold denotes the solutions that have the lowest LCOE values for the given wind farm sizes using the binary constraint set for the larger wind farm size resulted in the same layout presented by Grady et al. (2005), whereas for each of the other five cases, improvements were highlighted compared to the relevant reference case. As is highlighted in Table 3, for both wind farm sizes, the variation in costs as a result of the changes in layout are very small as the micrositing within the $4 \mathrm{~km}^{2}$ wind farm area results in very minimal changes in the installation costs. In fact, as the port position was not defined in the original case, it was necessary to place the port very far away relative to the size of the wind farm in order to remove any bias to the port's position. As a result of this, there are relatively large transit times to the wind farm included in each installation cost which are unaffected by the wind farm layout, but a function of the wind farm's distance from the installation port.

\subsection{Case 2: constant wind speed, variable direction}

The results for each of the constraint sets and wind farm sizes are summarized in Table 4 and the corresponding layouts are shown in Fig. 5. The original layouts proposed by the reference studies are shown in Fig. 3b, e. From Table 4, it can be seen that similar to the results for Case 1, the newly developed layout optimization framework for offshore wind farms is capable of identifying improvements using the PSO under all three constraint sets for both wind farm sizes.

\subsection{Case 3: variable wind speed, variable direction}

The results of executing the current framework with the PSO are found in Table 5 with the corresponding layouts plotted in Fig. 6 and the original reference layouts in Fig. 3c, f. Similar to the previous cases, the PSO using any of the constraint sets was capable of identifying improved layouts with regards to the LCOE. Similar to the previous cases, the best results were found using the array constraints.

\section{Discussion}

Using the present tool, cost variations as a result of changes to the wind farm layout are captured and included in the calculation of the layout's LCOE. For a small wind farm such as those considered here, it is, however, the increase in AEP which drives the decreases in LCOE, which is why for many cases an increase in lifetime cost is observed; however, the corresponding increase in AEP is sufficiently large to still result in a net reduction of the LCOE.

As would be expected, relaxing the turbine positioning constraints by designing arrays within the boundary or by treating the wind farm area as a continuous domain results in significant improvements in the LCOE as the shape of the layout can be designed to best utilize the characteristics of the site. Somewhat surprisingly, the continuous optimizer which represents the most unconstrained case was unable to consistently find improvements over the array optimizer. However, both were consistently able to find improvements compared to the binary optimizer which made use of the discretized wind farm area. Interestingly, the array optimizer appears more capable than the others to adjust the shape of the wind farm layout to take advantage of the wind resource.

As the array optimizer and continuous optimizer did not identify similar solutions it suggests that further tuning of the PSO is necessary in order to ensure that the optimizers are not prematurely converging to a local solution. Furthermore, given the results it indicates that moving from the binary or array optimizers to the continuous optimizer increases the size of the problem quite significantly. In the present case, all three constraint sets were solved using the same size of swarm; however, it might be more prudent for the swarm size to change depending on which constraint set is used thereby allowing the more complex problem to be solved with a larger swarm in order to avoid premature convergence. With a sufficiently large swarm, it should be possible for the PSO to converge to a higher quality solution closer to that of the global optimum. It should be 


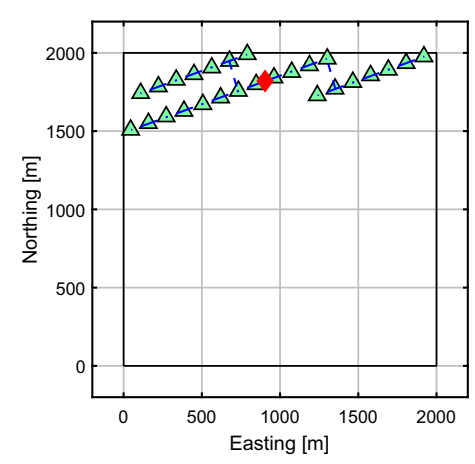

(a) 26 turbines - Array

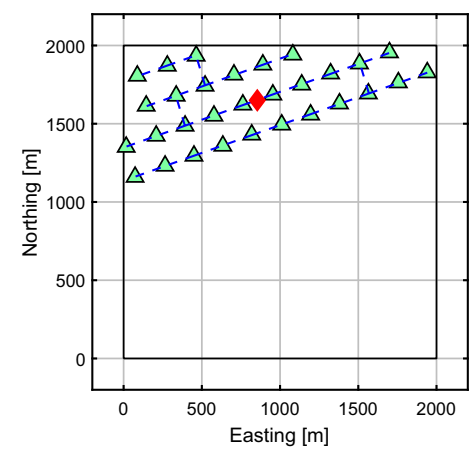

(d) 30 turbines - Array

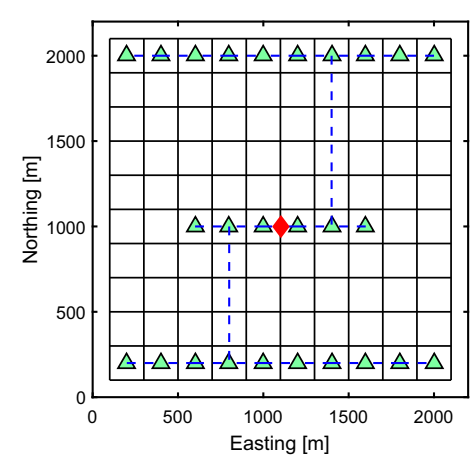

(b) 26 turbines - Binary

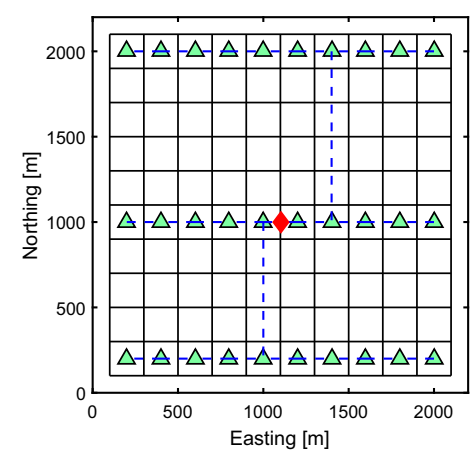

(e) 30 turbines - Binary

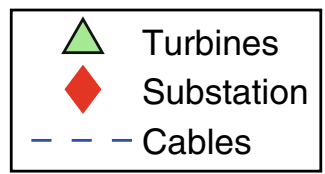

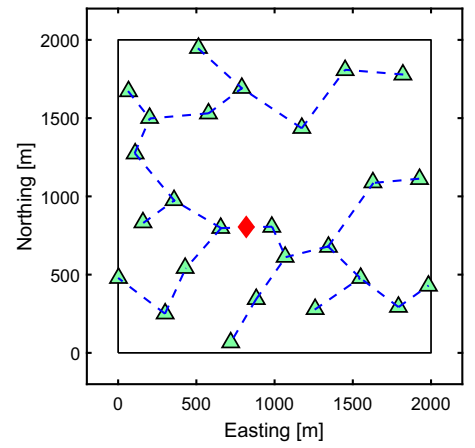

(c) 26 turbines - Continuous

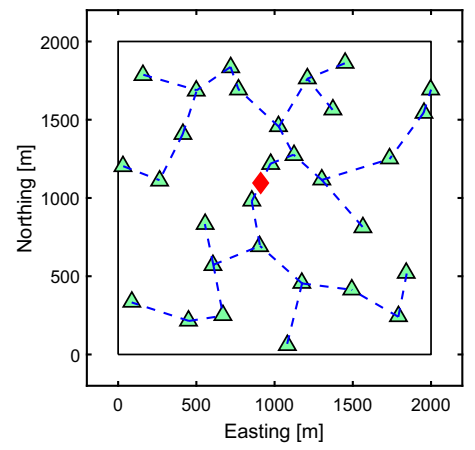

(f) 30 turbines - Continuous

Fig. 4 Optimized layouts for the case of a constant wind speed and constant direction with 26 turbines (top row) and 30 turbines (bottom row) using both optimization algorithms and all three constraint sets

Table 4 Layout optimization results: constant wind speed, variable direction

\begin{tabular}{lllll}
\hline Study & $\begin{array}{l}\text { Number of } \\
\text { turbines }\end{array}$ & Lifetime cost $(£)$ & AEP (MWh) & LCOE (£/MWh) \\
\hline Mosetti et al. (1994) & 19 & $3.77 \times 10^{8}$ & $8.17 \times 10^{4}$ & 540.25 \\
Array constraints & 19 & $3.77 \times 10^{8}$ & $8.32 \times 10^{4}$ & $\mathbf{5 3 0 . 7 9}$ \\
Binary constraints & 19 & $3.77 \times 10^{8}$ & $8.21 \times 10^{4}$ & 537.49 \\
Continuous constraints & 19 & $3.77 \times 10^{8}$ & $8.19 \times 10^{4}$ & 538.29 \\
Grady et al. (2005) & 39 & $5.62 \times 10^{8}$ & $1.57 \times 10^{5}$ & 419.13 \\
Array constraints & 39 & $5.61 \times 10^{8}$ & $1.61 \times 10^{5}$ & $\mathbf{4 0 8 . 0 7}$ \\
Binary constraints & 39 & $5.61 \times 10^{8}$ & $1.59 \times 10^{5}$ & 413.00 \\
Continuous constraints & 39 & $5.62 \times 10^{8}$ & $1.58 \times 10^{5}$ & 417.29
\end{tabular}

Bold denotes the solutions that have the lowest LCOE values for the given wind farm sizes noted, however, that metaheuristic algorithms like the PSO cannot guarantee, especially for a complex objective function such as the LCOE, that the optimization process will converge to the global optimum. Given the computational power allocated for this study, however, it was not possible to execute the optimizers with larger swarms. With swarms of 100 individuals as used in this study, each optimization took between one and three days to execute depending on the wind farm size and the selected constraints when executed on a desktop computer with an Intel Xeon 8- 


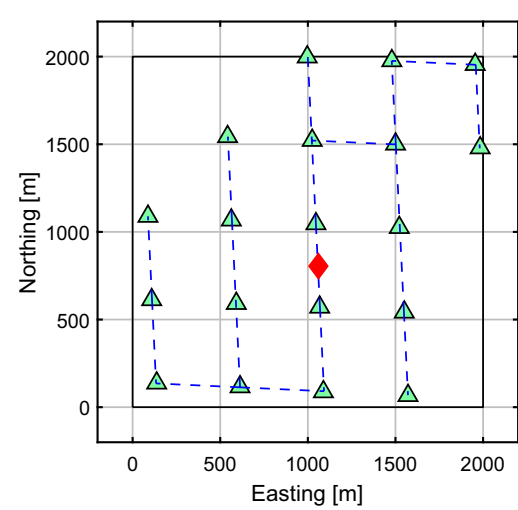

(a) 19 turbines - Array

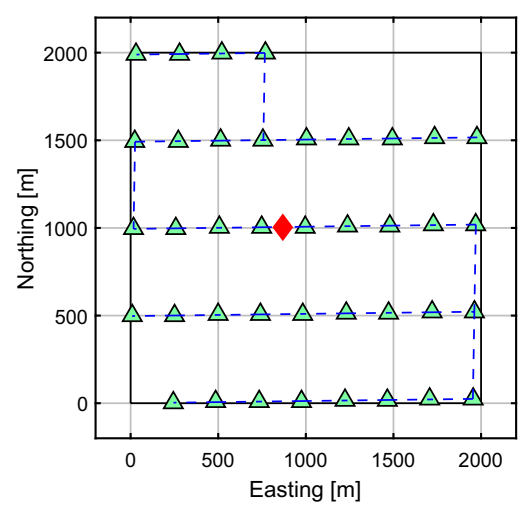

(d) 39 turbines - Array

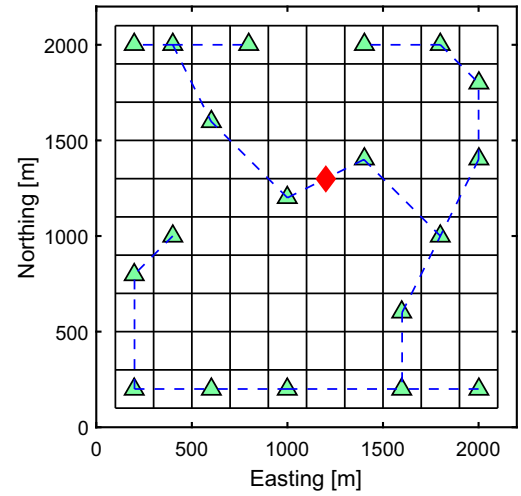

(b) 19 turbines - Binary

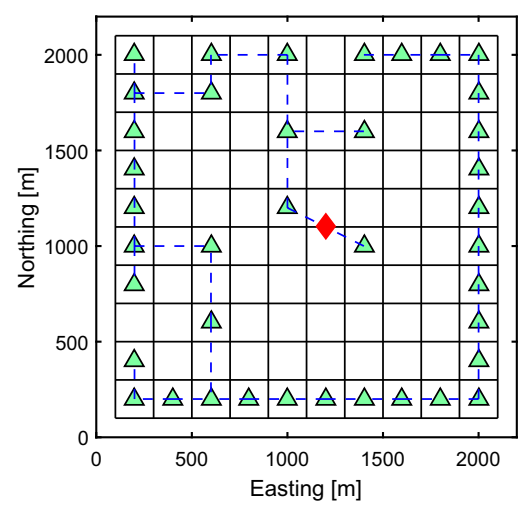

(e) 39 turbines - Binary

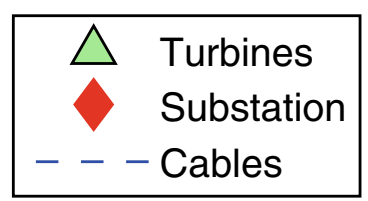

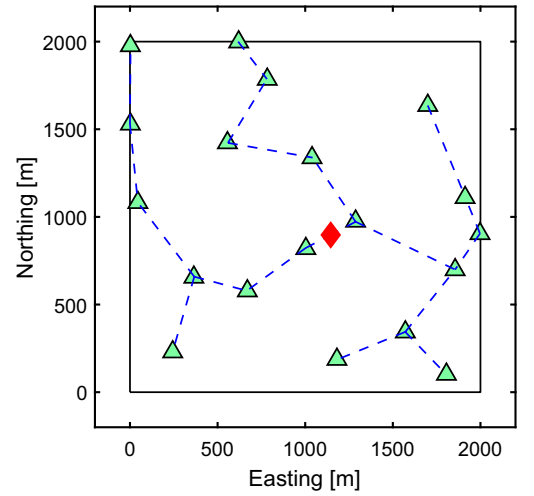

(c) 19 turbines - Continuous

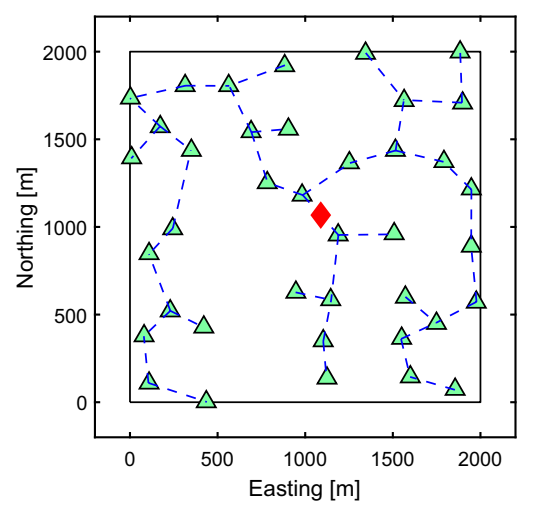

(f) 39 turbines - Continuous

Fig. 5 Optimized layout for the case of a constant wind speed and variable direction with 19 and 39 turbines using both optimization algorithms and all three constraint sets

Table 5 Layout optimization results: variable wind speed, variable direction

\begin{tabular}{lllll}
\hline Study & $\begin{array}{l}\text { Number of } \\
\text { turbines }\end{array}$ & Lifetime cost (£) & AEP (MWh) & LCOE (£/MWh) \\
\hline Mosetti et al. (1994) & 15 & $3.40 \times 10^{8}$ & $6.89 \times 10^{4}$ & 576.94 \\
Array constraints & 15 & $3.39 \times 10^{8}$ & $6.93 \times 10^{4}$ & $\mathbf{5 7 1 . 5 1}$ \\
Binary constraints & 15 & $3.39 \times 10^{8}$ & $6.91 \times 10^{4}$ & 573.87 \\
Continuous constraints & 15 & $3.39 \times 10^{8}$ & $6.91 \times 10^{4}$ & 574.22 \\
Grady et al. (2005) & 39 & $5.62 \times 10^{8}$ & $1.74 \times 10^{5}$ & 377.14 \\
Array constraints & 39 & $5.63 \times 10^{8}$ & $1.75 \times 10^{5}$ & $\mathbf{3 7 5 . 5 0}$ \\
Binary constraints & 39 & $5.62 \times 10^{8}$ & $1.75 \times 10^{5}$ & 376.72 \\
Continuous constraints & 39 & $5.62 \times 10^{8}$ & $1.75 \times 10^{5}$ & 376.72
\end{tabular}

Bold denotes the solutions that have the lowest LCOE values for the given wind farm sizes
CPU processor rated at $3.3 \mathrm{GHz}$. As the three different constraint sets lead to three different instances of the prob- lem with different decision variables, the design spaces are not directly comparable and each of the three optimizers 


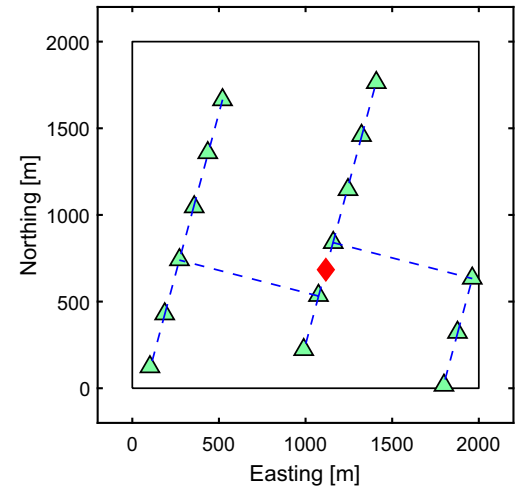

(a) 15 turbines - Array

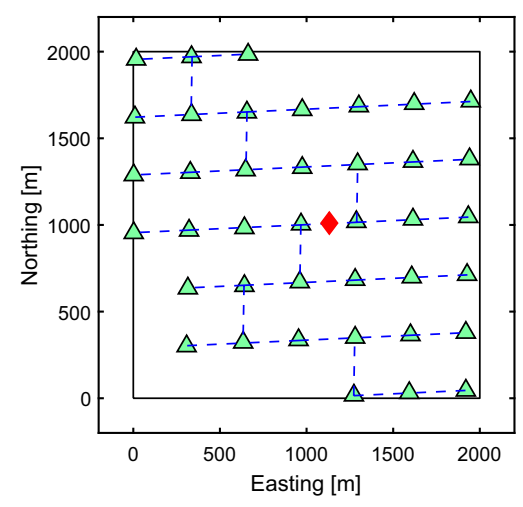

(d) 39 turbines - Array

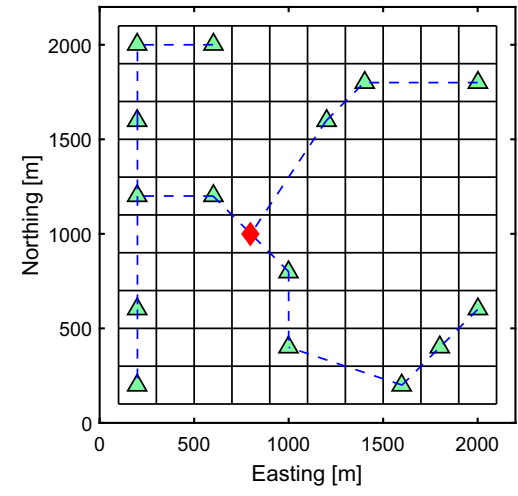

(b) 15 turbines - Binary

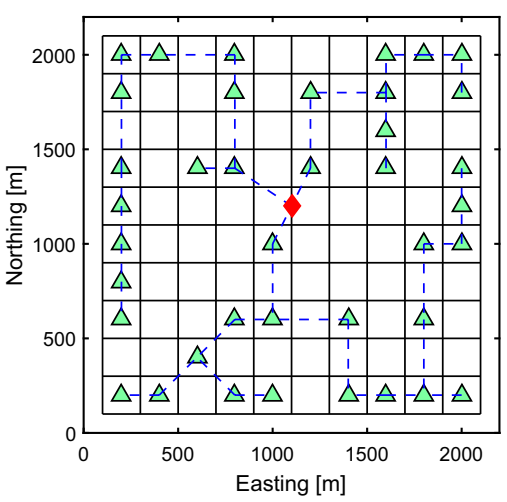

(e) 39 turbines - Binary

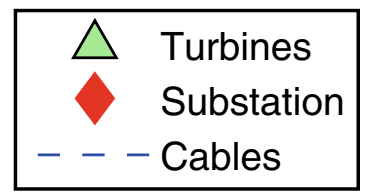

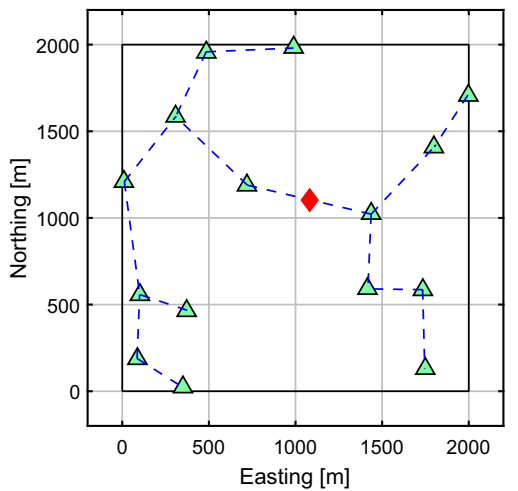

(c) 15 turbines - Continuous

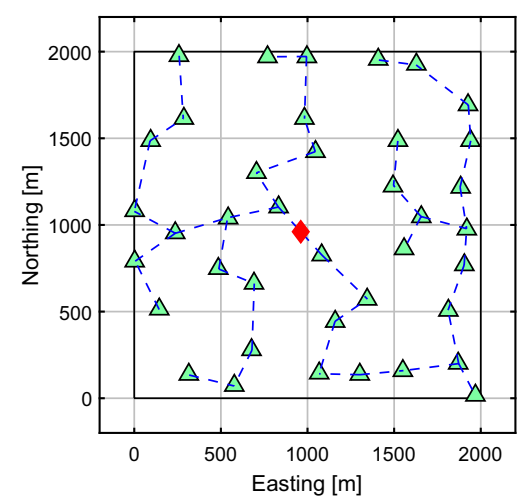

(f) 39 turbines - Continuous

Fig. 6 Optimized layout for the case of a variable wind speed and variable direction with 15 and 39 turbines using both optimization algorithms and all three constraint sets

should be tuned independently in order to ensure the best performance.

Looking at Case 1, it can be seen that both the binary and continuous optimizers use the majority of the available space, while the array optimizer is capable of identifying that it should sacrifice a close spacing in the direction perpendicular to the single wind direction. The binary optimizer is unable to find a similar solution due to the resolution of the discrete grid used in the binary optimization. This suggests that the discretization of the wind farm area should be done at a higher resolution to afford the optimizer a greater degree of flexibility. The present study used the 100 allowable turbine positions as this is what had been used in past studies. Increasing the number of allowable turbine positions through a higher resolution would, however, increase the size of the problem and potentially slow the rate of convergence.
The continuous optimizer should, however, be capable of identifying a similar solution, and the fact that it does not highlights that further work remains to be done with this optimizer in order to ensure that high quality solutions are reached.

The results from Case 2, however, indicate that the binary optimizer is placing more turbines on the edge of the wind farm in order to take advantage of the symmetrical wind resource, especially in the larger wind farm case. For this resource case and the larger wind farm, compared to the full continuous optimizer the binary optimizer results in better AEP values, demonstrating that additional constraints on the problem can reduce the search space without sacrificing the quality of the ultimate layouts.

Limiting the turbine positions to 100 possible positions significantly constrained the search space such that the 
solutions had inferior fitness values compared to the more relaxed constraint sets. This indicates that moving to the binary constraints with a discretized set of turbine positions over-constrains the problem, eliminating high-quality valid solutions. Considering the Mosetti cases, the impact of this on the LCOE varied from $£ 1$ to $£ 70$ per MWh increases, corresponding to $0-16 \%$ potential improvements in LCOE from relaxing the constraints. Given some of the assumptions, the percentage difference is smaller than it would be if this were a real site, as there are some fixed costs which are intentionally overestimated. As described earlier, the port location was defined as far away relative to the size of the wind farm in order to avoid the optimizer clustering turbines close to the installation port. The installation costs are, therefore, larger than they would be for a real case thereby increasing the LCOE. For these cases, it is, therefore, more valuable to analyse the absolute difference in LCOE rather than the percentage reduction.

Interestingly, Case 3 which represents the most realistic wind resource case finds very small variations in AEP across the three different constraint sets demonstrating that for a more varied wind speed and wind direction combinations all three constraint sets have merit and are capable of finding good solutions. The choice of which constraint set to use, therefore, becomes a function of what constraints are imposed on the site developer by consenting agencies or other stakeholders. The results from this case also demonstrate that there are several different layouts with similar AEP, cost, and LCOE values showing the complexity of the search space. Given that there are different layouts which can result in similar solutions the tuning of the optimizer becomes more important and further work will need to further explore this in order to ensure that the optimization process is not overlooking significant improvements and that the optimizer is operating in appropriate time scales.

\section{Conclusion}

This paper has presented the first results of an extended wind farm layout optimization framework making use of a more detailed LCOE evaluation function than existing layout optimization tools. This framework which makes use of a previously validated LCOE evaluation function has been applied to three different case studies using three different sets of placement constraints and two different wind farm sizes for each resource case in order to highlight both the applicability of a PSO given the increased detail and the improvements that can be made relative to the reference studies. The PSO applied to these three benchmark case studies have presented layouts with improved LCOE compared to past studies using a genetic algorithm. Furthermore, the results shown here indicate that the PSO is of interest to this area of research as the results can be obtained at a lower computational cost compared to a genetic algorithm.

By using multiple constraint sets it is also shown that by limiting the optimizer to create gridded layouts does not result in poor solutions, though the observed trends highlight the need for further tuning of the PSO in order to insure that the optimizer does not prematurely converge. Further work should explore both using multiple runs rather than single runs in order to avoid any seeding bias as well as using additional computational power thereby allowing larger swarms to be tested.

Acknowledgements This work is funded in part by the Energy Technologies Institute (ETI) and RCUK energy program for IDCORE (EP/J500847/1) and supported by EDF Energy R\&D UK Centre.

Open Access This article is distributed under the terms of the Creative Commons Attribution 4.0 International License (http://creativecomm ons.org/licenses/by/4.0/), which permits unrestricted use, distribution, and reproduction in any medium, provided you give appropriate credit to the original author(s) and the source, provide a link to the Creative Commons license, and indicate if changes were made.

\section{References}

Arthur D, Vassilvitskii S (2007) $k$-means++: the advantages of careful seeding. In: Proceedings of the 18th annual ACM-SIAM symposium on discrete algorithms, New Orleans, vol 8, pp 1-11

Barthelmie RJ, Folkerts L, Larsen GC, Frandsen ST, Rados K, Pryor SC, Lange B, Schepers G (2006) Comparison of wake model simulations with offshore wind turbine wake profiles measured by Sodar. J Atmos Ocean Technol 23(7):888-901. https://doi.org/10. 1175/JTECH1886.1

Barthelmie RJ, Hansen K, Frandsen ST, Rathmann O, Schepers JG, Schlez W, Phillips J, Rados K, Zervos A, Politis ES, Chaviaropoulos PK (2009) Modelling and measuring flow and wind turbine wakes in large wind farms offshore. Wind Energy 12(5):431-444. https://doi.org/10.1002/we.348

Bauer J, Lysgaard J (2015) The offshore wind farm array cable layout problem-a planar open vehicle routing problem. J Oper Res Soc 66(3):1-16. http://www.ii.uib.no/ joanna/papers/owfacl.pdf

Burke EK, Kendall G (2013) Search methodologies, 2nd edn. Springer, Boston. https://doi.org/10.1007/0-387-28356-0

Chen Y, Li H, Jin K, Song Q (2013) Wind farm layout optimization using genetic algorithm with different hub height wind turbines. Energy Convers Manag 70:56-65. https://doi.org/10.1016/ j.enconman.2013.02.007. http://linkinghub.elsevier.com/retrieve/ pii/S0196890413000873

Chowdhury S, Tong W, Messac A, Zhang J (2012) A mixed-discrete particle swarm optimization algorithm with explicit diversitypreservation. Struct Multidiscip Optim 47(3):367-388. https://doi. org/10.1007/s00158-012-0851-Z

Chowdhury S, Zhang J, Messac A, Castillo L (2013) Optimizing the arrangement and the selection of turbines for wind farms subject to varying wind conditions. Renew Energy 52(315):273-282. https:// doi.org/10.1016/j.renene.2012.10.017. http://linkinghub.elsevier. com/retrieve/pii/S0960148112006544www.sciencedirect.com/ science/article/pii/S0960148112006544

Clerc M, Kennedy J (2002) The particle swarm—explosion, stability, and convergence in a multidimensional complex space. IEEE Trans Evolut Comput 6(1):58-73. https://doi.org/10.1109/4235.985692 
Couto TG, Farias B, Diniz ACGC, Morais MVGD (2013) Optimization of wind farm layout using genetic algorithm. In: 10th world congress on structural and multidisciplinary optimization, Orlando, pp 1-10

DNV GL_Energy (2014) WindFarmer theory manual. GL Garrad Hassan, Bristol. https://www.dnvgl.com/services/windfarmer-3766

DuPont BL, Cagan J (2012) An extended pattern search approach to wind farm layout optimization. J Mech Des134(8):081,002. https://doi.org/10.1115/1.4006997. http://mechanicaldesign. asmedigitalcollection .asme.org/article.aspx ?articleid $=1484782$

Dutta S, Overbye T (2013) A graph-theoretic approach for addressing trenching constraints in wind farm collector system design. In: 2013 IEEE power and energy conference at Illinois (PECI), Urbana, pp 48-52. https://doi.org/10.1109/PECI.2013. 6506033. http://ieeexplore.ieee.org/lpdocs/epic03/wrapper.htm? arnumber $=6506033$

Eberhart R, Kennedy J (1995) A new optimizer using particle swarm theory. In: MHS'95 proceedings of the sixth international symposium on micro machine and human science, pp 39-43. https://doi. org/10.1109/MHS.1995.494215

Eberhart RCR, Yuhui Shi, Shi Y (2001) Particle swarm optimization: developments, applications and resources. In: Proceedings of the 2001 congress on evolutionary computation (IEEE Cat No 01TH8546), vol 1, pp 81-86. https://doi.org/10.1109/CEC.2001. 934374. http://ieeexplore.ieee.org/lpdocs/epic03/wrapper.htm? arnumber=934374, http://ieeexplore.ieee.org/xpls/abs_all.jsp? arnumber $=934374$

Elkinton CN (2007) Offshore wind farm layout optimization. Doctor of Philosophy Dissertation, University of Massachussetts, Amherst

Elkinton CN, Manwell JF, McGowan JG (2008) Algorithms for offshore wind farm layout optimization. In: Wind engineering, pp 67-83. http://multi-science.metapress.com/index/ Y14XL29NU6565RP1.pdf

Fagerfjäll P (2010) Optimizing wind farm layout-more bang for the buck using mixed integer linear programming. Master of Science Dissertation, Chalmers University of Technology and Gothenburgh University

Feng J, Shen WZ (2015) Solving the wind farm layout optimization problem using random search algorithm. Renew Energy 78:182192. https://doi.org/10.1016/j.renene.2015.01.005

Feng J, Shen WZ, Xu C (2016) Multi-objective random search algorithm for simultaneously optimizing wind farm layout and number of turbines. J Phys Conf Ser 753(3):032,011. https://doi.org/10.1088/ $1742-6596 / 753 / 3 / 032011$

Gaillard H (2015) Optimization of export electrical infrastructure in offshore windfarms. Master of Science Thesis, KTH Industrial Engineering and Management

Gaumond M, Rethore P, Bechmann A (2012) Benchmarking of wind turbine wake models in large offshore windfarms. In: Proceedings of the science of making torque from wind conference, Oldenburg. http://www.eera-dtoc.eu/wp-content/uploads/files/ Gaumond-et-al-Benchmarking-of-wind-turbine-wake-modelsin-large-offshore-wind-farms.pdf

Gaumond M, Réthoré P, Ott S, Bechmann A, Hansen K (2013) Evaluation of the wind direction uncertainty and its impact on wake modeling at the Horns Rev offshore wind farm. Wind Energy. https://doi.org/10.1002/we.1625

Geem ZW (2013) Hong J (2013) Improved formulation for the optimization of wind turbine placement in a wind farm. Math Probl Eng 1:1-5. https://doi.org/10.1155/2013/481364. http:// www.hindawi.com/journals/mpe/2013/481364/

Grady S, Hussaini M, Abdullah M (2005) Placement of wind turbines using genetic algorithms. Renew Energy 30(2):259-270. https:// doi.org/10.1016/j.renene.2004.05.007. http://linkinghub.elsevier. com/retrieve/pii/S0960148104001867
Gurobi Optimization Inc (2015) Gurobi optimizer reference manual. http://www.gurobi.com. Accessed 5 Jan 2015

Hassan R, Cohanim B, de Weck O (2005) A comparison of particle swarm optimization and the genetic algorithm. In: 1st AIAA multidisciplinary design optimization specialist conference, pp 1-13. https://doi.org/10.2514/6.2005-1897

Hou P, Hu W, Soltani M, Chen C, Chen Z (2017) Combined optimization for offshore wind turbine micro siting. Appl Energy 189:271-282. https://doi.org/10.1016/j.apenergy.2016.11.083

Huang HS (2009) Efficient hybrid distributed genetic algorithms for wind turbine positioning in large wind farms. In: IEEE international symposium on industrial electronics (ISIE), pp 2196-2201. http://ieeexplore.ieee.org/xpls/abs_all.jsp?arnumber=5213603

IEC (2006) IEC 60228: conductors of insulated cables, 3rd edn. International Electrotechnical Commission, Geneva

IEC (2006) IEC 60287: electric cables-calculation of the current rating-Part 1-1: Current rating equations (100\% load factor) and calculation of losses-general, 2nd edn. International Electrotechnical Commission, Geneva

Ituarte-Villarreal CM, Espiritu JF (2011) Optimization of wind turbine placement using a viral based optimization algorithm. Proc Comput Sci 6:469-474. https://doi.org/10.1016/j.procs.2011.08.087

Kennedy J, Eberhart R (1995) Particle swarm optimization. In: IEEE international conference on neural networks, 1995, Perth, vol 4, pp 1942-1948. https://doi.org/10.1109/ICNN.1995.488968

Larsen GC (1988) A simple wake calculation procedure. In: Technical report, Risø National Laboratory. Report No. Risø-M-2760

Lindahl M, Bagger NF, Stidsen T, Ahrenfeldt SF, Arana I (2013) OptiArray from DONG energy. In: Proceedings of the 12th wind integration workshop (international workshop on large-scale integration of wind power into power systems as well as on transmission networks for offshore wind power plants), London

Makridis A, Chick J (2013) Validation of a CFD model of wind turbine wakes with terrain effects. J Wind Eng Ind Aerodyn 123:12-29. https://doi.org/10.1016/j.jweia.2013.08.009

Marmidis G, Lazarou S, Pyrgioti E (2008) Optimal placement of wind turbines in a wind park using Monte Carlo simulation. Renew Energy 33(7):1455-1460. https://doi.org/10.1016/ j.renene.2007.09.004. http://linkinghub.elsevier.com/retrieve/pii/ S0960148107002807

Mittal A (2010) Optimization of the layout of large wind farms using a genetic algorithm. Master of Science Dissertation, Case Western Reserve University

Mosetti G, Poloni C, Diviacco B (1994) Optimization of wind turbine positioning in large wind-farms by means of a genetic algorithm. $\mathrm{J}$ Wind Eng Ind Aerodyn 51(1):105-116. http://www.sciencedirect. com/science/article/pii/0167610594900809

NOREL Group (2014) Nautical and offshore renewable energy Liaison Group (NOREL) minutes of the 29th NOREL held on 8 May 2014. In: Technical report, The Crown Estate

Pillai AC, Chick J, de Laleu V (2014) Modelling wind turbine wakes at Middelgrunden wind farm. In: Proceedings of European wind energy conference \& exhibition 2014, Barcelona, pp 1-10

Pillai A, Chick J, Johanning L, Khorasanchi M, Pelissier S (2016a) Optimisation of offshore wind farms using a genetic algorithm. Int J Offshore Polar Eng 26(3):225-234. https://doi.org/10.17736/ ijope.2016.mmr16

Pillai AC, Chick J, Johanning L, Khorasanchi M, Barbouchi S (2016) Comparison of offshore wind farm layout optimizaiton using a genetic algorithm and a particle swarm optimizer. In: Proceedings of the ASME 2016 35th international conference on ocean, offshore and arctic engineering (OMAE 2016), ASME, Busan, vol 6, pp 1-11. https://doi.org/10.1115/OMAE2016-54145

Pillai AC, Chick J, Johanning L, Khorasanchi M, de Laleu V (2015) Offshore wind farm electrical cable layout optimization. Eng Optim 47(12):1689-1708. https://doi.org/10. 
1080/0305215X.2014.992892. www.scopus.com/inward/record. url?eid=2-s2.0-84922374336\&partnerID=tZOtx3y 1

Pookpunt S, Ongsakul W (2013) Optimal placement of wind turbines within wind farm using binary particle swarm optimization with time-varying acceleration coefficients. Renew Energy 55:266276. https://doi.org/10.1016/j.renene.2012.12.005

Renkema DJ (2007) Validation of wind turbine wake models. Master of Science Dissertation, TU Delft

Réthoré PE, Fuglsang P, Larsen TJ, Buhl T, Larsen GC (2011) TOPFARM wind farm optimization tool. Riso DTU National Laboratory for Sustainable Energy, Roskilde

Rodrigues S, Restrepo C, Katsouris G, Teixeira Pinto R, Soleimanzadeh M, Bosman P, Bauer P (2016) A multi-objective optimizaiton framework for offshore wind farm layouts and electric infrastructures. Energies 9(3):1-42. https://doi.org/10.3390/en9030216

Shakoor R, Yusri M, Raheem A, Rasheed N (2016) Wind farm layout optimization using area dimensions and definite point selection techniques. Renew Energy 88:154-163. https://doi.org/10.1016/j. renene.2015.11.021

Tegen S, Hand M, Maples B, Lantz E, Schwabe P, Smith A (2012) 2010 cost of wind energy review. In: Technical report, National Renewable Energy Laboratory (NREL). Report No. NREL/TP5000-52920

Tegen S, Lantz E, Hand M, Maples B, Smith A, Schwabe P (2013) 2011 cost of wind energy review. In: Technical report, National Renewable Energy Laboratory. Report No. NREL/TP-5000-56266
Thøgersen ML (2005) windPRO/PARK. EMD International A/S, Aalborg. http://www.emd.dk

Van Den Bergh F, Engelbrecht AP (2006) A study of particle swarm optimization particle trajectories. Inf Sci 176(8):937-971. https:// doi.org/10.1016/j.ins.2005.02.003

Wan C, Wang J, Yang G, Zhang X (2010) Optimal micro-siting of wind farms by particle swarm optimization. Lect Notes Comput Sci 2. https://doi.org/10.1007/978-3-642-13495-1_25

Wan C, Wang J, Yang G, Zhang X (2010) Particle swarm optimization based on Gaussian mutation and its application to wind farm micro-siting. In: 49th IEEE conference on decision and control (CDC) (July 2015), pp 2227-2232. https://doi.org/10.1109/CDC. 2010.5716941

Zhang PY (2013) Topics in wind farm layout optimisation: analytical wake models, noise propagation, and energy production. Master of Applied Science Dissertation, University of Toronto

Zhang PY, Romero DA, Beck JC, Amon CH (2014) Solving wind farm layout optimization with mixed integer programs and constraint programs. EURO J Comput Optim 2(3):195-219. https://doi.org/ 10.1007/s13675-014-0024-5 